\title{
Vibration Noises and Acoustic Optimization Design of the Centrifugal Pump
}

\author{
Zhanqi $\mathrm{HaO}^{1, \text { a }}$ \\ ${ }^{1}$ Water and power supply service center of North China oil field, Renqiu, Hebei, China, 062552 \\ a296947111@qq.com
}

Keywords: centrifugal pump; vibration; noises; optimization design

\begin{abstract}
In daily production and life, the centrifugal pump plays an important role, but its vibration noise has influenced humans' mind and physical health. This paper adopts the vibration noise of the centrifugal pump as the research objective, analyzing how to reduce noises. The focus of this paper is on the exposition of the vibration noises of the centrifugal pump and the optimization design, and the purpose is to reduce the influence of such noises.
\end{abstract}

\section{Introduction}

In recent years, vibration noises have drawn wide attention. As a hot research issue, structural acoustic optimization provides reliable methods for treatment of vibration noises of the centrifugal pump. In order to give full play to the method and effectively solve the issue of the centrifugal pump's vibration, this paper introduces the overall situation of structural acoustic optimization, analyzes the inner flow field and noise optimization treatment of the centrifugal pump, aiming at improving design and reducing noises of the centrifugal pump.

\section{Overview of noise reduction}

\section{Significance.}

With the development of people's living standards, factors influencing people's mental and physical health have been in the spotlight. Noises disturb people's ordinary work, life and learning on the one hand; on the other hand, impede people's thinking, communication and rest. Worse still, they might cause accidents, doing a great harm to people's mental and physical health. Therefore, noise control is very imperative. With the rapid development of science, noise pollution is worsening. In order to effectively reduce noises, various countries have intensified their efforts to treat noise pollution, and put forward different control methods.

\section{Noise control methods.}

Currently, there are three methods to control noises, namely controlling the sound source, cutting off the noise transmission approaches and transferring the major sound source into mechanical power. The first method has been proved to be the most effective, which can be further divided into active control and passive control. The former refers to reducing vibration through the excitation source; the latter refers to improving the structure to reduce vibration. This paper mainly studies passive control, and employs the structural acoustic optimization method to change the form, the size and other aspects of the structure so as to achieve effective control of vibration noises.

The structural acoustic optimization method can effectively reduce noises through analysis, calculation and discussion of noises. Made up of structural design, computer application and optimization algorithm, the method can ensure design quality [1], but the method is still in the preliminary development period. In the face with complex vibration structures, the method should pay attention to the following aspects: ensuring the accuracy of the model construction; applying the simplified model to a relatively narrow frequency band scope and improving the modification measures to ensure the effective transformation of the method; emphasizing on studying stiffness distribution and application of compound materials. 


\section{Vibration noises and acoustic optimization of the centrifugal pump}

\section{Analysis of the inner flow field of the centrifugal pump.}

Vibration of the centrifugal pump is influenced by multiple factors. The most critical factor is the radial force, which is caused by the rotating impeller. As a core part of the centrifugal pump, the impeller suffers disproportional pressure, leading to the emergence of the radial force. In order to reduce the vibration noises, it is necessary to analyze the inner flow of the impeller so as to learn the distribution of the inner speed and inner pressure of the centrifugal pump. According to the current research data, the author found out that, though there are many literatures about the inner flow of the centrifugal pump, most of them focus on analyzing the flowing speed instead of the inner radial force. Therefore, the vibration noises of the centrifugal pump have not yet been efficiently reduced or eliminated.

Based on the author's study, the vibration noises of the centrifugal pump have a close bearing on pressure fluctuation and eddies caused by instable flows. In order to control noises induced by inner flow, the following measures can be taken: 1) Optimize the space interval between the tongue and the impeller: Though the method can control vibration noises, it has obvious flaws, in that, once the space interval between the two exceeds $20 \%$ the waterpower will be decreased during noise reduction; 2 ) Adjust the number of impeller blades: Since the number of impeller blades is related to waterpower loss, energy loss and vibration noises, certain amount of waterpower and energy might be lost during noise reduction. Therefore, the number of blades should be suitable to practical situations; 3) Control the noise sound: Vibration noises of the centrifugal pump might not be influenced by the asymmetric liquid flows, so the inhibition of the liquid flows can achieve the goal of reducing vibration noises [2].

\section{Acoustic optimization of the centrifugal pump.}

During the practical optimization process, the pedestal is adopted as a research object. The specific optimization measures include thickening of the pedestal and enhancing its stiffness so s to reduce the noise radiation.

At present, research into optimized control of noises focused on simple structures, such as boards, beams and poles. Optimization measures, including mass change, partial modification and rectangular flatting, are adopted. In recent years, optimized control of noises of engineering structures is still in the preliminary stage. Some foreign scholars have built the engine's dynamic model, designed relevant variables, etc. However, there have not yet been reports about optimized control of noises. The major reason for the research gap is that research into optimized control of noises is extremely difficult. The research difficulty is mainly reflected in two aspects. On the one hand, during optimization, as the variables change, the system model should be continuously adjusted to enhance the reconstruction times and difficulty of relevant models; on the other hand, the prediction accuracy of noises and the speed are conflicted. The problem is especially prominent in terms of complex structures.

Since the acoustic optimization issue is a complex and difficult one, the author made some preparations, such as building and modifying the finite element model or boundary element model, choosing the suitable optimization methods, ensuring the prediction efficiency of the noise radiation, etc. The acoustic radiation power of the centrifugal pump before and after improvement is compared and analyzed. Results suggest that the thickening of the pedestal can significantly abate vibration noises of the centrifugal pump [3].

\section{Conclusions}

To sum up, the worsening noise pollution has directly influenced people's mental and physical health. In order to efficiently control noises and reduce harms thus incurred, this paper adopts vibration noises of the centrifugal pump as a research object, discusses the significance and methods to reduce noises, and focuses on expounding methods to reduce vibration noises through the inner flow of the centrifugal pump and measures to optimize noise treatment of the centrifugal pump. The 
author is convinced that the issue of vibration noises of the centrifugal pump can be efficiently solved by following the method put forward in this paper.

\section{References}

[1] J. van der Geer, J.A.J. Hanraads, R.A. Lupton, The art of writing a scientific article, J. Sci. Commun. 163 (2000) 51-59.

Reference to a book:

[2] W. Strunk Jr., E.B. White, The Elements of Style, third ed., Macmillan, New York, 1979.

Reference to a chapter in an edited book:

[3] G.R. Mettam, L.B. Adams, How to prepare an electronic version of your article, in: B.S. Jones, R.Z. Smith (Eds.), Introduction to the Electronic Age, E-Publishing Inc., New York, 1999, pp. 281-304.

[4] R.J. Ong, J.T. Dawley and P.G. Clem: submitted to Journal of Materials Research (2003)

[5] P.G. Clem, M. Rodriguez, J.A. Voigt and C.S. Ashley, U.S. Patent 6,231,666. (2001)

[6] Information on http://www.weld.labs.gov.cn 